\title{
Relationship between infectious gastroenteritis and irritable bowel syndrome
}

\author{
Motoyori Kanazawa $\cdot$ Shin Fukudo
}

Received: 28 November 2013/ Accepted: 1 December 2013/Published online: 15 December 2013

(C) Springer Japan 2013

\begin{abstract}
A newly recognized causative factor in a subset of patients with irritable bowel syndrome (IBS) is called post-infectious IBS (PI-IBS). IBS symptoms frequently develop after an acute episode of infectious gastroenteritis. Several studies have been made in our understanding of the concept of PI-IBS. Recent studies suggest that transient or chronic gastrointestinal inflammation may play a role in IBS pathogenesis. PI-IBS can be regarded as a natural experiment model in which an insult in the form of an infective disease impacts on subjects with underlying genetic and psychosocial predispositions who then develop IBS. IBS is likely to be a complex trait wherein variability in clinical presentation is partially explained by heterogeneity in underlying genetic and environmental risk factors for PI-IBS. Further studies on PI-IBS are needed to understand the pathophysiology of functional gastrointestinal disorders and to develop new promising management for such patients.
\end{abstract}

Keywords Irritable bowel syndrome (IBS) - Infectious gastroenteritis - Risk factors - Mucosal microscopic inflammation

\section{Introduction}

One of major functional gastrointestinal disorders (FGIDs), irritable bowel syndrome (IBS), is defined as a gastrointestinal disorder in which abdominal pain or discomfort is

M. Kanazawa $(\bowtie) \cdot$ S. Fukudo

Department of Behavioral Medicine, Tohoku University

Graduate School of Medicine, 2-1 Seiryo, Aoba,

Sendai 980-8575, Japan

e-mail: m-kanazawa@med.tohoku.ac.jp associated with defecation or a change in bowel habit, and with features of disordered defecation, for which there is no apparent structural basis [1]. The prevalence of IBS is 10-20\% and most studies indicate a female predominance in Western and Eastern countries [1,2].

Bowel habit subtypes in IBS are based on dominant stool consistency according to current Rome III diagnostic criteria [3]. IBS with diarrhea (IBS-D) is classified by loose (mushy) or watery stools (i.e., Bristol Stool Form Scale 1-2, Table 1) $>25 \%$ and hard or lumpy stool (i.e., Bristol Stool Form Scale 6-7, Table 1) $<25 \%$ of bowel movements. On the other hand, IBS with constipation (IBS-C) is classified by hard or lumpy stool (i.e., Bristol Stool Form Scale 6-7, Table 1) $>25 \%$ and loose (mushy) or watery stools (i.e., Bristol Stool Form Scale 1-2, Table 1) $<25 \%$ of bowel movements. The classification system has been important especially in clinical trials, where a positive effect could be expected only in subgroups of patients [3]. However, the bowel habits in IBS patients show considerable inter- and intra-individual variability.

A newly recognized causative factor in a subset of IBS patients is post-infectious IBS (PI-IBS). Subjects diagnosed with IBS are more likely to have an episode of infectious gastroenteritis compared to non-IBS subjects [4]. IBS symptoms frequently develop after an acute episode of infectious gastroenteritis [5]. In the current article, associations between infectious gastroenteritis and functional (non-organic) gastrointestinal disorders, especially IBS, are discussed, focusing on the new concept of PI-IBS.

\section{Epidemiology of PI-IBS}

It has been apparent for decades that patients who develop infectious gastroenteritis are at an increased risk for FGIDs. 
Table 1 The Bristol Stool Form Scale (reference [3])

\begin{tabular}{ll}
\hline Type & Description \\
\hline 1 & Separate hard lumps like nuts (difficult to pass) \\
2 & Sausage shaped but lumpy \\
3 & Like a sausage but with cracks on its surface \\
4 & Like a sausage or snake, smooth and soft \\
5 & Soft blobs with clear-cut edges (passed easily) \\
6 & Fluffy pieces with ragged edges, a mushy stool \\
7 & Watery, no solid pieces \\
\hline
\end{tabular}

Table 2 Definition of post-infectious IBS (reference [5])

1. Acute onset of new IBS symptoms after an episode of acute infectious gastroenteritis

2. Following an acute illness characterized by $\geq 2$ of the following:
(a) Fever
(b) Vomiting
(c) Diarrhea
(d) A positive stool culture

In 1962, Chaudhary and Truelove [6] reported that 34 of 130 IBS patients had an episode of history of dysentery with severe diarrhea. Despite this evidence, the potential relationship between infectious gastroenteritis and IBS was neglected until the past decade. Since then, several studies have been made in our understanding of the concept of PIIBS in Western and Eastern countries [5].

Recent studies suggest that transient or chronic gastrointestinal inflammation may play a role in IBS pathogenesis. PI-IBS is defined as the acute onset of new IBS symptoms in an individual who has not previously met the Rome criteria for IBS, immediately following an acute illness characterized by $\geq 2$ of the following-fever, vomiting, diarrhea, or a positive bacterial stool culture (Table 2) [5]. The majority (approximately two-thirds) of cases of PI-IBS meet the Rome criteria for IBS-D [7].

The incidence of PI-IBS has been reported to vary between $3.7 \%$ and $36 \%$ [5]. A meta-analysis investigated eight studies revealed that the median incidence of IBS in all patients with a previous episode of infectious gastroenteritis was $9.8 \%$ (median duration of follow-up was 9 months), whereas the incidence was $1.2 \%$ in control subjects [8]. Recently, a significant association between FGIDs and infectious gastroenteritis was identified using medical encounter data from the US military [9]. The risk for functional diarrhea was most clearly elevated (odds ratio 6.28) and the risk of IBS was following (odds ratio 3.72). The risk of functional constipation and functional dyspepsia showed moderate associations (odds ratio 2.15 and 2.39, respectively). Therefore, subjects who had an episode of infectious gastroenteritis have a significantly increased risk for developing not only IBS but also the other functional gastrointestinal disorders compared to uninfected subjects.

\section{Risk factors and pathophysiology of PI-IBS}

A number of risk factors have been identified for PI-IBS (Table 3). It has been investigated that biologic risk factors for persistent of IBS after an episode of bacterial gastroenteritis were female gender; existence of abdominal pain, fever, or bloody stools during the acute infection; diarrhea $>6$ days; and a peak stool frequency $>10$ /day [10]. Thabane et al. [11] confirmed important predictors for development of PI-IBS using the risk model. In another study, smoking was also found to be a risk factor of PI-IBS [12]. Interestingly, older age ( $>60$ years) appears to be associated with a protective effect [13]. Specific pathogens may also influence the risk of developing PI-IBS. Infection due to Campylobacter or Shigella species may induce more severe mucosal damage in the gastrointestinal tract and may lead to longer duration of acute illness compared with infection due to Salmonella species [13]. Recently, it has been reported that viral gastroenteritis due to norovirus infection leads to the development of IBS in a substantial proportion of patients, which is similar to that reported after bacterial gastroenteritis [14]. Although biological factors are certainly important in the genesis of PI-IBS, psychological factors may also play an important role. It has been confirmed that pre-existing anxiety or depression, hypochondriasis, or adverse life events in the previous year

Table 3 Risk factors for post-infectious IBS

1. Female gender

2. Younger age

3. Severity of the gastrointestinal insult during acute infection
(a) Persistent diarrhea
(b) Peak stool frequency
(c) Abdominal pain
(d) Bloody stools
(e) Fever
(f) Body weight loss
(g) Infection with Campylobacter or Shigella species

4. Psychosocial factors
(a) Anxiety
(b) Depression
(c) Somatization
(d) Adverse life events

5. Smoking

6. Gene variant (TLR9, IL6, CDH1) 
was a strong risk for development of PI-IBS [10, 15]. There is a clear episode in time and well-defined pathological changes in the gut before the onset in patients who are considered to be PI-IBS. Therefore, PI-IBS can be regarded as a natural experiment model in which an insult in the form of an infective disease impacts on subjects with underlying genetic and psychosocial predispositions who then develops IBS.

\section{Gut mucosal changes and increased intestinal permeability in PI-IBS}

Although endoscopic mucosal findings or conventional histological examinations of mucosal biopsy in patients with IBS show no abnormality, some of mucosal changes have been observed when detailed quantification is performed. Current evidence for PI-IBS suggests the persistence of mucosal microscopic inflammation in the gut from patients who go on to develop persistent IBS symptoms. Spiller et al. [16] found patients who develop PI-IBS show increased numbers of lymphocytes, T lymphocytes, and macrophages in various compartments of the small bowel and colon. Others have reported an increased number of mast cells in the terminal ileum of these patients [17]. It has been proposed that inflammatory mediators produced by mast cells (e.g., histamine, proteases and serotonin) and $\mathrm{T}$ cells (e.g., cytokines) affect enteric and extrinsic nerves as well as smooth muscle activity leading to bowel habit changes and abdominal pain [18]. Furthermore, it has been investigated that serine protease release by luminal bacteria or mucosal mast cells, which are increased in IBS-D could result from increased membrane permeability and pain sensitivity via protease-activated receptor 2 (PAR-2) [19]. Another important abnormality was detected in PI-IBS that relates to increased numbers of serotonin-containing enterochromaffin (EC) cells [7]. Serotonin (5-HT) receptors in the gut, especially subtypes of $5-\mathrm{HT}_{3}$ and $5-\mathrm{HT}_{4}$ receptors, are considered to play an important role on gastrointestinal motility, pain sensitivity and membrane permeability, and the efficacy of some serotonin receptor modulators has been investigated in patients with IBS [20]. It has been demonstrated that increases in both inflammatory cells and enteroendocrine cells (e.g., EC cells) were found in patients with PI-IBS. There was a good correlation between numbers of inflammatory cells and enteroendocrine cells [16], suggesting that cytokines might drive enteroendocrine hyperplasia.

Patients with PI-IBS demonstrate increased small intestinal permeability compared to non-IBS controls, suggesting a defect in epithelial integrity that might promote intestinal inflammation [16]. Increased intestinal permeability can promote inflammation by facilitating exposure of submucosa to luminal antigens with subsequent disturbance of gut sensitivity and motility. In addition, it has been reported that an acute psychological stressor increases small intestinal permeability in a subset of healthy subjects without intestinal gastroenteritis [21]. It is interesting to note that rectal hypersensitivity and colon transit were similar in patients with and without PI-IBS following intestinal infection; even though PI-IBS patients have elevated mucosal inflammatory cell counts, those who did not also develop IBS symptoms (i.e., non-PI-IBS) [15]. The similarity of the pathophysiology shown in PI-IBS and nonPI-IBS patients suggests that prior gut infection is unlikely to be a primary cause of abnormal colon transit and visceral sensitivity in IBS.

On the other hand, there is evidence that genetic risk factors may contribute to PI-IBS pathophysiology (Table 3). A recent study identified three candidate gene variants, namely Toll-like receptor-9 (TLR9), cadherin-1 $(C D H 1)$, interleukin-6 (ILO), which were associated with development of IBS following acute gastroenteritis [22]. These results suggest that PI-IBS might result from abnormalities in gene encoding epithelial barrier functions and innate immune responses to enteric bacteria. This is consistent with a paradigm of PI-IBS pathogenesis involving decreased mucosal barrier function and abnormal immune activation in the gut mucosa. Further studies are needed to confirm whether patients with PI-IBS show specific features of associations between gene variant and physiological findings in the gut when compared to sporadic patients with IBS.

\section{Prognosis and management of PI-IBS}

Prognosis of PI-IBS has not been studied systematically. Some studies have suggested that the symptoms persist for years and then gradually improve, possibly with a better prognosis compared with IBS of noninfectious origin [10]. In two long-term follow-up studies, less than half of cases with PI-IBS recovered at 5-6 years [23, 24]. Furthermore, in the largest prospective cohort study, the prevalence of PI-IBS in subjects with a history of dysentery in Walkerton, Ontario in Canada dropped from $28.3 \%$ at 2-3 years to $21.4 \%$ at 4 years, $14.3 \%$ at 6 years and $15.4 \%$ at 8 years [10]. After 8 years, the incidence of new-onset IBS was no longer increased among subjects who were exposed to the initial infection when compared to those who were not. Thus, overall prognosis is considered to be good over an infectious period of 5-6 years.

It is important that patients with PI-IBS should understand the roles of stress, life style (including diet), psychological symptoms and persisting low-grade inflammation in their condition. Potential differential condition should also 
be considered as infection can unmask other gastrointestinal disorders such as inflammatory bowel disease, diverticular disease and celiac disease. Currently, there are no specific treatment options for PI-IBS. Management for PI-IBS should be adjusted to the severity of the patient's symptoms which is similar to that for IBS in general. Not only abdominal pain but also urgency and diarrhea are main concerns for IBS patients with diarrhea. Loperamide or codeine which affects prolongation of intestinal transit [25] can be useful to prevent fecal urgency and diarrhea associated with IBS. Low doses of tricyclic antidepressants (e.g., amitriptyline) may also help to reduce visceral pain sensitivity and slow intestinal transit for patients with IBS-D [26]. Serotonergic agents have been confirmed to be modestly effective for IBS symptoms. $5-\mathrm{HT}_{3}$ receptor antagonists (e.g., alosetron) enhance small intestinal absorption, slow colonic transit and may reduce visceral pain sensitivity in diarrhea-predominant patients [27]. On the other hand, 5- $\mathrm{HT}_{4}$ receptor agonists (e.g., tegaserod), accelerate orocecal transit and increase the frequency of bowel movements, and they been used for constipation-predominant IBS [28]. However, these agents have never been tested specifically in patients with PI-IBS.

It has been reported that patients with IBS are likely to have small intestinal bacterial overgrowth (SIBO) [29]. The prevalence of SIBO in patients with PI-IBS has not yet been evaluated. Dietary change can influence the composition of the gut microbiota and consequently reduce IBS symptoms. Restriction of fermentable oligo-, di-, monosaccharides, and polyols (FODMAP) diet has been demonstrated to be beneficial in some subsets of patients with IBS [30]. Although the role of probiotics or antibiotics in the treatment of IBS is still controversial, these agents may be considered in those patients with a positive breath test result. Meta-analysis of previous controlled trials has shown that probiotics were significantly better than placebo [31]. Nonabsorbable antibiotics (e.g., rifaximin) have recently demonstrated efficacy for IBS symptoms [32]. Anti-inflammatory agents such as mesalazine and mast cell stabilizers such as ketotifen are also expected to be another potential therapeutic option for PI-IBS [33, 34]. Further high-quality randomized controlled trials are needed to confirm efficacy of these treatments for patients with PIIBS.

\section{Future directions}

The pathophysiology of IBS remains incompletely understood. Patients with PI-IBS may be associated with less psychological abnormalities and have a better prognosis than other IBS patients; however, these findings are still controversial. In fact, several psychological aspects are identified as being risk factors for development of PI-IBS. IBS is likely to be a complex trait wherein variability in clinical presentation is partially explained by heterogeneity in underlying genetic and environmental risk factors for PIIBS. It is important to investigate the mechanisms that underlie the clinical/pathological features in PI-IBS since the patients are a more homogenous group and show dominant diarrhea symptoms when compared to sporadic IBS. The more novel findings observed in PI-IBS may help us to understand the complex pathophysiology of IBS and the other functional gastrointestinal disorders such as functional diarrhea and to develop new promising treatments for such patients.

\section{Disclosures}

Conflict of Interest: Kanazawa and Fukudo declare that they have no conflict of interest.

Human/Animal Rights: All procedures followed were in accordance with the ethical standards of the responsible committee on human experimentation (institutional and national) and with the Helsinki Declaration of 1975, as revised in 2008(5).

Informed Consent: This study does not apply to giving Informed Consent because the present manuscript is a review article.

\section{References}

1. Drossman DA, Camilleri M, Mayer EA, Whitehead WE. AGA technical review on irritable bowel syndrome. Gastroenterology. 2002;123:2108-31.

2. Gwee KA, Lu CL, Ghoshal UC. Epidemiology of irritable bowel syndrome in Asia: something old, something new, something borrowed. J Gastroenterol Hepatol. 2009;24:1601-7.

3. Longstreth GF, Thompson WG, Chey WD, et al. Functional bowel disorders. Gastroenterology. 2006;130:1480-91.

4. Kanazawa M, Endo Y, Whitehead WE, et al. Patients and nonconsulters with irritable bowel syndrome reporting a parental history of bowel problems have more impaired psychological distress. Dig Dis Sci. 2004;49:1046-53.

5. Spiller R, Garsed K. Postinfectious irritable bowel syndrome. Gastroenterology. 2009;136:1979-88.

6. Chaudhary NA, Truelove SC. The irritable colon syndrome. A study of the clinical features, predisposing causes, and prognosis in 130 cases. Q J Med. 1962;31:307-22.

7. Dunlop SP, Jenkins D, Neal KR, Spiller R. Relative importance of enterochromaffin cell hyperplasia, anxiety, and depression in postinfectious IBS. Gastroenterology. 2003;125:1651-9.

8. Halvorson HA, Schlett CD, Riddle MS. Postinfectious irritable bowel syndrome-a meta-analysis. Am J Gastroenterol 2006;101:1894-9.

9. Porter CK, Gormley R, Tribble DR, et al. The incidence and gastrointestinal infectious risk of functional gastrointestinal disorders in a healthy US adult population. Am J Gastroenterol. 2011;106:130-8.

10. Marshall JK, Thabane M, Garg AX, et al. Eight year prognosis of postinfectious irritable bowel syndrome following waterborne bacterial dysentery. Gut. 2010;59:605-11.

11. Thabane M, Simunovic M, Akhtar-Danesh N, Marshall JK. Development and validation of a risk score for post-infectious 
irritable bowel syndrome. Am J Gastroenterol. 2009;104: 2267-74.

12. Parry SD, Barton JR, Welfare MR. Factors associated with the development of post-infectious functional gastrointestinal diseases: does smoking play a role? Eur J Gastroenterol Hepatol. 2005; 17:1071-5.

13. Neal KR, Hebden J, Spiller RC. Prevalence of gastrointestinal symptoms six months after bacterial gastroenteritis and risk factors for development of the irritable bowel syndrome: postal survey of patients. Br Med J. 1997;314:779-82.

14. Zanini B, Ricci $C$, Bandera F, et al. Incidence of post-infectious irritable bowel syndrome and functional intestinal disorders following a water-borne viral gastroenteritis outbreak. Am J Gastroenterol. 2012;107:891-9.

15. Gwee KA, Leong YL, Graham C, et al. The role of psychological and biological factors in postinfective gut dysfunction. Gut. 1999;44:400-6.

16. Spiller RC, Jenkins D, Thornley JP, et al. Increased rectal mucosal enteroendocrine cells, $\mathrm{T}$ lymphocytes, and increased gut permeability following acute Campylobacter enteritis and in post-dysenteric irritable bowel syndrome. Gut. 2000;47:804-11.

17. Lee KJ, Kim YB, Kim JH, et al. The alteration of enterochromaffin cell, mast cell, and lamina propria $\mathrm{T}$ lymphocyte numbers in irritable bowel syndrome and its relationship with psychological factors. J Gastroenterol Hepatol. 2008;23:1689-94.

18. Barbara G, Stanghellini V, De Giorgio R, et al. Activated mast cells in proximity to colonic nerves correlate with abdominal pain in irritable bowel syndrome. Gastroenterology. 2004;126:693-702.

19. Cenac N, Andrews CN, Holzhausen M, et al. Role for protease activity in visceral pain in irritable bowel syndrome. J Clin Invest. 2007;117:636-47.

20. Gershon MD, Tack J. The serotonin signaling system: from basic understanding to drug development for functional GI disorders. Gastroenterology. 2007;132:397-414.

21. Vanuytsel T, van Wanrooy S, Vanheel H, et al. Psychological stress and corticotropin-releasing hormone increase intestinal permeability in humans by a mast cell-dependent mechanism. Gut. (Epub ahead of print).

22. Villani AC, Lemire M, Thabane M, et al. Genetic risk factors for post-infectious irritable bowel syndrome following a waterborne outbreak of gastroenteritis. Gastroenterology. 2010;138:1502-13.
23. Neal KR, Barker L, Spiller RC. Prognosis in post-infective irritable bowel syndrome: a six year follow up study. Gut. 2002;51:410-3.

24. Jung IS, Kim HS, Park H, Lee SI. The clinical course of postinfectious irritable bowel syndrome: a five-year follow-up study. J Clin Gastroenterol. 2009;43:534-40.

25. Szilagyi A, Salomon R, Seidman E. Influence of loperamide on lactose handling and oral-caecal transit time. Aliment Pharmacol Ther. 1996;10:765-70.

26. Morgan V, Pickens D, Gautam S, Kessler R, Mertz H. Amitriptyline reduces rectal pain related activation of the anterior cingulate cortex in patients with irritable bowel syndrome. Gut. 2005;54:601-7.

27. Delvaux M, Louvel D, Mamet JP, Campos-Oriola R, Frexinos J. Effect of alosetron on responses to colonic distension in patients with irritable bowel syndrome. Aliment Pharmacol Ther. 1998;12:849-55.

28. Harish K, Hazeena K, Thomas V, Kumar S, Jose T, Narayanan P. Effect of tegaserod on colonic transit time in male patients with constipation-predominant irritable bowel syndrome. J Gastroenterol Hepatol. 2007;22:1183-9.

29. Ford AC, Spiegel BM, Talley NJ, Moayyedi P. Small intestinal bacterial overgrowth in irritable bowel syndrome: systematic review and meta-analysis. Clin Gastroenterol Hepatol. 2009;7: 1279-86.

30. Halmos EP, Power VA, Shepherd SJ, et al. A diet in FODMAPs reduces symptoms of irritable bowel syndrome. Gastroenterology. (Epub ahead of print).

31. McFarland LV, Dublin S. Meta-analysis of probiotics for the treatment of irritable bowel syndrome. World J Gastroenterol. 2008;14:2650-61.

32. Pimentel M. Review of rifaximin as treatment for SIBO and IBS Expert Opin Investig Drugs. 2009;18:349-58.

33. Corinaldesi R, Stanghellini V, Cremon C, et al. Effect of mesalazine on mucosal immune biomarkers in irritable bowel syndrome: a randomized controlled proof-of-concept study. Aliment Pharmacol Ther. 2009;30:245-52.

34. Klooker TK, Braak B, Koopman KE, et al. The mast cell stabiliser ketotifen decreases visceral hypersensitivity and improves intestinal symptoms in patients with irritable bowel syndrome. Gut. 2010;59:1213-21. 